\title{
Video Article \\ Analysis of Learning and Memory Ability in an Alzheimer's Disease Mouse Model using the Morris Water Maze
}

\author{
Huiling Tian ${ }^{1}$, Ning Ding ${ }^{2}$, Mengwei Guo ${ }^{1}$, Shun Wang ${ }^{1}$, Zidong Wang ${ }^{1}$, Hao Liu ${ }^{1}$, Jiayi Yang ${ }^{1}$, Yujie Li ${ }^{3}$, Jingyu Ren ${ }^{1}$, Jing Jiang $^{4}$, Zhigang Li $^{1}$ \\ ${ }^{1}$ School of Acupuncture-Moxibustion and Tuina, Beijing University of Chinese Medicine \\ ${ }^{2}$ Guang'anmen Hospital of China Academy of Chinese Medical Science \\ ${ }^{3}$ The Third Affiliated Hospital of Beijng University of Chinese Medicine \\ ${ }^{4}$ School of Nursing, Beijing University of Chinese Medicine
}

Correspondence to: Jing Jiang at yingxi7847@126.com, Zhigang Li at Lizhigang620@126.com

URL: https://www.jove.com/video/60055

DOI: doi:10.3791/60055

Keywords: Behavior, Issue 152, Morris water maze, protocol, behavior, Alzheimer' Disease, mouse, manual acupuncture

Date Published: 10/29/2019

Citation: Tian, H., Ding, N., Guo, M., Wang, S., Wang, Z., Liu, H., Yang, J., Li, Y., Ren, J., Jiang, J., Li, Z. Analysis of Learning and Memory Ability in an Alzheimer's Disease Mouse Model using the Morris Water Maze. J. Vis. Exp. (152), e60055, doi:10.3791/60055 (2019).

\section{Abstract}

A Morris water maze (MWM) experiment forces experimental animals to swim and learn to find a platform hidden in the water. It is widely used in scientific research to assess the learning and memory of animals. Due to the extensive use of the MWM test, visual experimental protocols are essential for researchers. This manuscript uses the latest studies to introduce the protocol of the MWM test. Alzheimer' Disease (AD) is characterized by a progressive loss of memory and cognitive function. An alternative and complementary treatment used for $A D$ is Manual Acupuncture (MA). To assess the learning and memory ability of AD model mice, the MWM test was conducted. The visible platform trial, hidden platform trial, probe trial, and reversal trial of MWM were used to evaluate spatial learning and memory ability. In the visible platform trial, the swimming speed and escape latency of mice in different groups was not significantly different. In the hidden platform and reversal trials, the AD group showed a long escape latency. The escape latency decreased significantly after the MA treatment. Low platform crossover number and the proportion of time in the SW quadrant in the probe trial increased after the MA treatment $(p<0.05$ or $p<0.01)$. The results of the MWM tests suggest that MA can effectively improve the spatial learning and memory abilities of AD model mice. Rigorous experimental operations provided assurance of the reliability of the results.

\section{Video Link}

The video component of this article can be found at https://www.jove.com/video/60055/

\section{Introduction}

Currently, the MWM experiment has become the most widely used and the standard behavioral experiment to evaluate the spatial learning and memory of animals ${ }^{1}$. It was initially designed by the British psychologist Richard G. Morris and has constantly been improved. Many advantages such as minimal training, cross-species utility, insensitivity to differences in body weight, and repeated testing ability of MWM make it the best method for assessing cognitive function ${ }^{2}$. Alzheimer's disease $(A D)$ is a major medical problem, primarily characterized by a decline in memory processing and cognitive function ${ }^{3}$. MWM is an indispensable experimental means to evaluate the learning and memory ability of $A D$ model animals and the effectiveness of intervention methods. MWM experiments are generally time consuming (6-11 days) and involve many variable factors ${ }^{4}$. Although there are many articles about water maze experiments, in practice, researchers lack a coherent protocol. Therefore, an intuitive and rigorous protocol process video is particularly important. Using a previous experiment as an example ${ }^{5}$, all the steps of the MWM are described. Using MWM, previous studies suggested that acupuncture could relieve the symptoms of AD model mice ${ }^{5,6,7}$.

Herein, the MWM protocol used in a recent study ${ }^{5}$ is described to provide a simple and visible method for researchers to assess the spatial learning and memory of AD model animals.

\section{Protocol}

This protocol was approved by the Animal Ethics Committee of Beijing University of Chinese Medicine, and it was in accordance with all guidelines for the Care and Use of Laboratory Animals of China. There was no accidental death situation during the experimental procedure, and no animals needed to be euthanized in this study.

\section{Preparation}

1. Purchase 30 male SAMP 8 mice and 10 male SAMR1 mice (age: 8 months).

2. House the mice individually in individual ventilation cages at a temperature of $24 \pm 2{ }^{\circ} \mathrm{C}$ and a $12 \mathrm{~h}$ dark/light cycle. 
3. Feed the mice with a standard pellet diet available ad libitum and provide sterile drinking water.

4. Acclimate all the mice to the environment for 5 days before experimentation.

\section{Grouping of animals}

1. Randomly divide 30 SAMP8 mice into three groups ( $n=10 /$ group): the AD group, manual acupuncture (MA) group, and medicine (M) group.

2. Use 10 SAMR1 mice as the normal control $(\mathrm{N})$ group $^{6}$.

\section{Administration of donepezil hydrochloride tablets}

1. Crush a donepezil hydrochloride tablet ( $5 \mathrm{mg} / \mathrm{tablet}$ ) and dissolve it in $50 \mathrm{~mL}$ of distilled water.

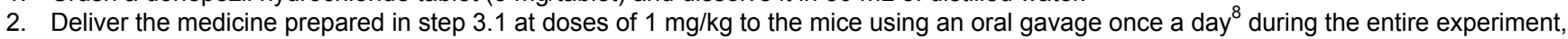
including the days when the MA treatment and MWM tests are performed.

\section{Administration of manual acupuncture}

1. Immobilize the mice of the MA group in mouse bags.

2. Use disposable sterile acupuncture needles $(0.25 \mathrm{~mm} \times 13 \mathrm{~mm})$ and apply the flat thorn method of MA on Baihui (GV20) and Yintang $(\mathrm{GV} 29)^{5}$ toward the nose for $20 \mathrm{~min}$. Ensure that the needle depth is $0.2-0.3 \mathrm{~cm}$.

3. Bidirectionally twirl the manipulation within $90^{\circ}$ at a speed of about $180 \mathrm{r} / \mathrm{min}$ every $5 \mathrm{~min}$ for $\sim 15 \mathrm{~s}$ each time during the entire experiment, including days when MA treatment and MWM tests are performed.

\section{MWM test}

NOTE: At $24 \mathrm{~h}$ after the 15 consecutive days of treatment, subject the mice in the four groups to the MWM test. Conduct the visible platform trial, hidden platform trial, probe trial, and reversal trial in order.

1. Prepare for the MWM test.

1. Position MWM device and the signal acquisition and processing system in an experiment room designed to maintain sound insulation.

2. Put a circular white tank (diameter $=90 \mathrm{~cm}$, height $=50 \mathrm{~cm}$ ) surrounded by an opaque cloth in the middle of the MWM device.

3. Fix a video camera to the ceiling of the MWM device and connect it to a video recorder with an automated tracking system to collect the data.

4. Divide the water maze tank equally into four equal regions using two mutually perpendicular lines, labeled north (N), south (S), east (E), and west (W). Divide the pool area conceptually into four quadrants of the same size (NE, NW, SW, and SE).

5. Within the sight of the mouse, place visual cues of different shapes on the wall of each quadrant as visual references (e.g., squares, triangles, and circles).

NOTE: Distal cues are the animal's navigational reference points for locating the platform. Therefore, do not move them during the test The position of the researcher is a potential distal cue and can influence the MWM. Therefore, the researcher should stay out of the sight of the mice while waiting for the animal to perform the test.

6. Fill the circular tank with water to a depth of $30 \mathrm{~cm}$ and maintain at $22 \pm 2{ }^{\circ} \mathrm{C}$ with an electric heater.

7. Render the water opaque with about $150 \mathrm{~g}$ of milk powder.

2. Perform the visible platform trial.

1. Place a plastic circular platform (diameter $=9.5 \mathrm{~cm}$; height $=28 \mathrm{~cm}$ ) $1 \mathrm{~cm}$ above the water surface in any quadrant at random.

2. Put a black flag on the platform.

3. Release each mouse gently into the water at water-level from one of the four start locations facing the tank wall. Do not drop the mouse into the water.

4. Activate the computer tracking program as soon as the mouse is released into the water.

5. Give each mouse $60 \mathrm{~s}$ to search for the platform. At the end of each trial, place each mouse on the platform and allow it to stay on it for 10-30 s.

6. Observe the swimming trajectories of the mice on the computer, record the time the mouse took to find the platform as escape latency, and analyze the swimming speed.

7. Dry each mouse with towels and warm it with an electric heater. Be sure to use an appropriate heat source to prevent the animal from overheating.

NOTE: Place each mouse into the pool at each of the four different starting quadrants for four trials, moving the platform to a different location with each subsequent trial. The interval between two trials using each mouse is 15-20 min.

3. Perform the hidden platform trial/place navigation test

1. Place the same platform without a flag in the SE quadrant.

2. Randomly place the mouse into the pool from each of the four quadrants (NE, NW, SW, N) facing the pool wall for four trials. Use a time interval of 15-20 min between two trials.

3. Give each mouse $60 \mathrm{~s}$ to search for the hidden platform.

4. Record the escape latency of each trial after the mouse climbs up to the platform for subsequent analysis.

5. Dry each mouse with towels and warm it with an electric heater.

NOTE: Conduct the hidden platform trial from days 2-6. If the mouse cannot find the platform in $60 \mathrm{~s}$, lead the mouse to climb up to the platform and allow it to stay there for 10-30 s at the end of each trial. Perform four trials/day for each mouse for 5 consecutive days, with the platform and the visual cues at constant positions. 
4. Perform the probe trial.

NOTE: Locate each mouse in the pool at a novel start position to observe the spatial exploration ability of the mouse.

1. Remove the platform.

2. Locate each mouse facing the tank wall in the pool once for $60 \mathrm{~s}$. Ensure that the starting location is the NW quadrant, which is the quadrant furthest away from the SE quadrant.

3. Record the swimming distance, swimming speed, and the platform crossover number in the maze.

4. Dry each mouse with towels and provide warmth after the trial.

5. Perform the reversal trial.

NOTE: Perform the reversal trial from days 8-11.

1. Position the platform in the middle of the NW quadrant (instead of the SE quadrant).

2. Follow steps 5.3.2 -5.3 .5 as detailed in the hidden platform trial section.

\section{Statistical Analysis}

1. Use statistics software (e.g., SPSS 20.0) to perform the statistical analysis.

\section{Representative Results}

The time axis diagram of this protocol is shown in Figure 1.

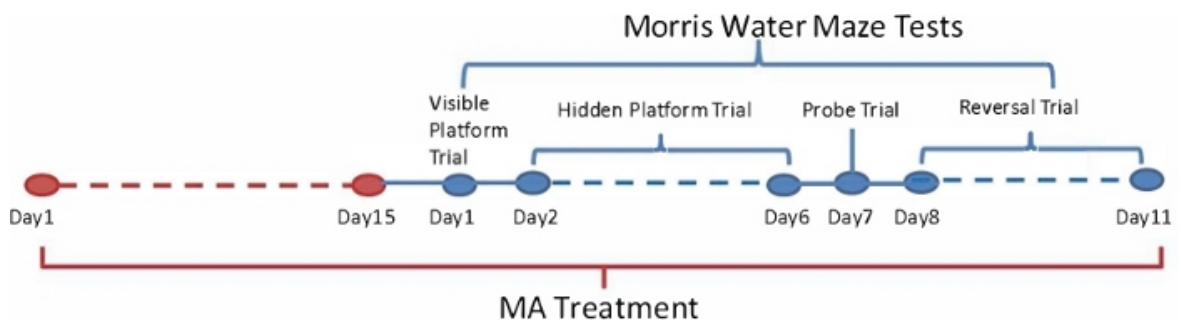

Figure 1: Time axis diagram of the study protocol. Please click here to view a larger version of this figure.

The time axis shows that this experiment lasted for a total of 21 days. The treatment was applied to the mouse during the whole experiment and the MWM tests began after 15 days of treatment. The visible platform, hidden platform, probe, and reversal trials were conducted in order.

Previously published results from Ding et al. ${ }^{5}$ are presented as typical results of MWM Figure 2. 
A Visible Platform Trial
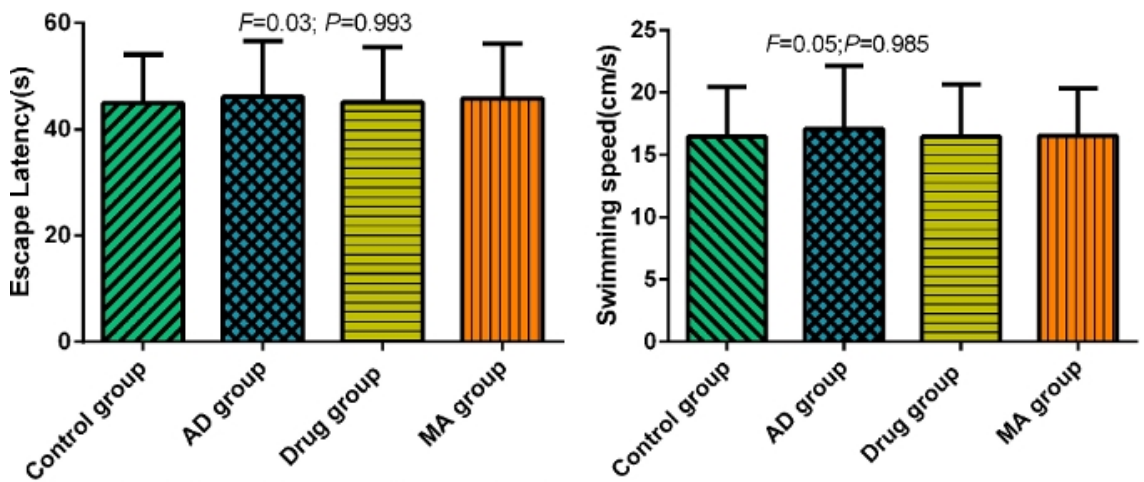

B Hidden Platform Trial and Reversal Trial

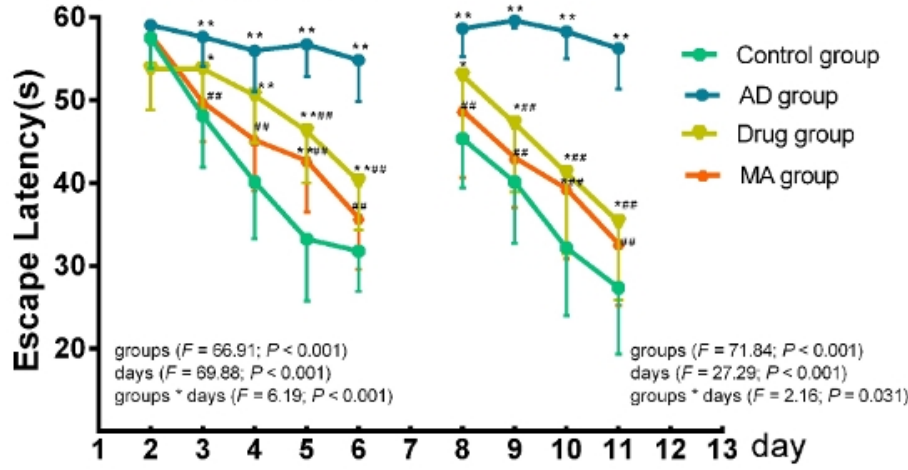

C Probe Trial
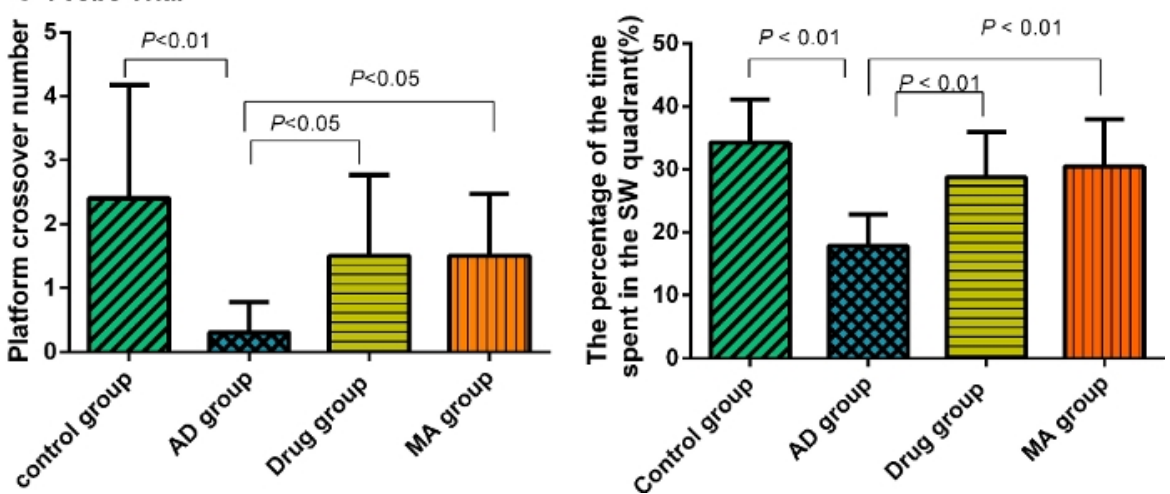

Figure 2: Typical results of the Morris water maze test $(n=10)$. (A) Changes in the escape latency and swimming speed of rats among the different groups in the visible platform trial. (B) Changes in the escape latency of rats among the different groups in the hidden platform and reversal trials. The $p$-values are ${ }^{*} p<0.05$ and ${ }^{* *} p<0.01$ compared with the control group. The symbol \#\# indicates $p<0.01$ compared with the AD group. (C) Changes in the platform crossover number and the percentage of the time spent by the rats in the northwest quadrant among the different experimental groups in the probe trial. The results of the visible platform, hidden platform, and reversal trial in each group are shown ( $\mathrm{n}$ $=10$, mean $\pm S D$ ). This figure has been modified from Ding et $\mathrm{l}^{5}$. Please click here to view a larger version of this figure.

Figure 2A shows the results of the visible platform trial. No statistical differences were observed in the escape latency or swimming speed among the groups on the first day of MWM. Figure 2B shows the results of the hidden platform and reversal trial from days 2-6 and days 8-11. The escape latency of the AD group remained at a high level on each day of the test. The escape latency of the other three groups decreased gradually. The escape latency from days 3-6 and days 8-11 was longer in the AD group than in the control group $(p<0.01)$. The escape latencies of mice in the MA and drug groups were shorter than that of mice in the AD group on days 2-6 and days 8-11, respectively ( $p<0.01)$. Figure 2C shows the results of the probe trial. The platform crossover number of mice in the AD group was statistically lower than that in the control group $(p<0.01)$. The platform crossover number in the MA group was higher than that in the AD group $(p<0.05)$. The proportion of time spent in the SW quadrant by mice in the AD group was significantly lower than that in the control group $(p<0.01)$. The proportion of time spent in the SW quadrant in the MA group was higher than that in the AD group $(p<0.01)$.

\section{Discussion}

Although many water mazes, including the Biel water maze and the Cincinnati water maze, have been around for at least a century, only the MWM has been widely used to effectively and objectively evaluate spatial learning and memory ability because it has many advantages ${ }^{9}$. Despite extensive use of the MWM, the procedure has not always been used optimally. MWM experiments generally take a long time and are 
influenced by many variable factors. There are some effective and reliable aspects that help detect changes in spatial learning and memory ability that should be taken into consideration.

Four different MWM trials were performed. The visible platform trial was used on day 1 of MWM. If the animals could swim directly to the platform, it indicated that the swimming ability and vision of the animals were normal ${ }^{10}$. Otnass suggested that the visible platform trial should be conducted first ${ }^{11}$. The results of the visible platform trial in this study meant that the four groups started at the same learning level. From there, the successive experiments could be started. The hidden platform trial was used to assess the ability of the mice to acquire learning and memory ability. The probe trial was conducted on day $7,24 \mathrm{~h}$ after the end of the hidden platform trial, to assess working memory. Finally, the reversal trial was used to assess working memory. The changes in the four different trials of MWM together indicated that the AD model mice had low learning and memory ability and that MA had a positive effect on $A D^{5}$.

There are no specific standards for the dimensions of the pool and platform ${ }^{1}$. A 214-cm diameter pool is used in most MWM studies. Vorhees and Williams demonstrated that with identical protocols, rats learn faster in a 122-cm pool than in a $210-\mathrm{cm}$ pool; the steep slope of the learning curve indicates that the $122-\mathrm{cm}$ diameter pool is extremely easy for rats to navigate ${ }^{12}$. In the current protocol, considering the old age and weak stature of the $A D$ mice, a $90-\mathrm{cm}$ diameter pool and $9.5-\mathrm{cm}$ diameter platform were used. The results of preliminary experiments indicated that mice had more difficulty finding the platform in a bigger diameter pool. Therefore, tests in larger pools do not represent the real difference among the groups. The experimental animals had a harder time finding the platform in a bigger pool with a smaller platform ${ }^{4}$. Therefore, the size of the pool and the platform must be optimized in preliminary experiments according to the experimental requirements and condition of the experimental animals.

Water at temperature ranging from $20-24{ }^{\circ} \mathrm{C}$ is recommended for performing the MWM test ${ }^{4}$. Aged experimental animals performed poorly in cold water ${ }^{13}$, indicating a clear age-dependent loss of thermoregulation ${ }^{14}$. In this study, a thermostat was placed at the bottom of the pool to maintain the temperature of the water at $20-24^{\circ} \mathrm{C}$. The study results showed no significant difference in the swimming speeds among the four groups ${ }^{5}$.

MWM is a powerful technique to assess cognitive function and is widely used in studies presently. However, there is no defined, standard, consistent equipment to perform the MWM test, including the sizes of the pool and platform ${ }^{15,16}$. Different laboratories have different specifications for MWM. Therefore, researchers choose the appropriate experimental device according to their individual experimental requirements, which may cause confusion among researchers. Preliminary experiments are also necessary. More studies need to be conducted on basic experiments like MWM. Presently, the flexibility of MWM as an experimental tool only lies in the ability to choose the basic protocols according to the study purposed. Therefore, this test can be applied to assess cognitive function in greater depth.

\section{Disclosures}

The authors declare no potential conflict of interest.

\section{Acknowledgments}

Huiling Tian and Ning Ding are co-first authors. Zhigang Li and Jing Jiang are co-corresponding authors. This research was supported by grants from the National Natural Science Foundation of China (Grant Nos. 81804178, 81473774, and 81503654). The protocol and results described herein originates from the article, "Involvement of Manual Acupuncture Regulates Behavior and Cerebral Blood Flow in the SAMP8 Mouse Model of Alzheimer's Disease" by Dr. Ning Ding et al.

\section{References}

1. Vorhees, C. V., Williams, M. T. Value of water mazes for assessing spatial and egocentric learning and memory in rodent basic research and regulatory studies. Neurotoxicology Teratology. 45, 75-90 (2014).

2. Vorhees, C. V., Williams, M. T. Morris water maze: procedures for assessing spatial and related forms of learning and memory. Nature Protocol. 1 (2), 848-58 (2006).

3. Alzheimer's Disease International. The state of the art of dementia research: New frontiers; World Alzheimer Report 2018. 9, 1-46 (2018).

4. Vorhees, C. V. et al. Effects of neonatal (+)-methamphetamine on path integration and spatial learning in rats: effects of dose and rearing conditions. International Journal of Developmental Neuroscience. 26 (6), 599-610 (2008).

5. Ding, N., Jiang, J., Xu, A., Tang, Y., Li, Z. Manual acupuncture regulates behavior and cerebral blood flow in the SAMP8 mouse model of Alzheimer's disease. Frontiers in Neuroscience. 13, 37 (2019).

6. Ding, N. et al. Manual acupuncture suppresses the expression of proinflammatory proteins associated with the NLRP3 inflammasome in the hippocampus of SAMP8 mice. Evidence-Based Complementary and Alternative Medicine. 2017, 1-8 (2017).

7. Cao, J. et al. Behavioral changes and hippocampus glucose metabolism in APP/PS1 transgenic mice via electro-acupuncture at governor vessel acupoints. Frontiers in Aging Neuroscience. 9, 5 (2017).

8. Amy, E. et al. Effects of sub-chronic donepezil on brain Abeta and cognition in a mouse model of Alzheimer's disease. Psychopharmacology. 230, 279-289 (2013).

9. Garthe, A., Kempermann, G. An old test for new neurons: refining the Morris water maze to study the functional relevance of adult hippocampal neurogenesis. Frontiers in Neuroscience. 7, 63 (2013).

10. Schoenfeld, R., Schiffelholz, T., Beyer, C., Leplow, B., Foreman, N. Variants of the Morris water maze task to comparatively assess human and rodent place navigation. Neurobiology of Learning and Memory. 139, 117-127 (2017).

11. Otnass, M. K., Brun, V. H., Moser, M., Moser, E. I. Pretraining prevents spatial learning impairment after saturation of hippocampal long-term potentiation. Journal of Neuroscience. 19 (24), 49 (1999).

12. Vorhees, C. V., Williams, M. T. Assessing spatial learning and memory in rodents. Ilar Journal. 55 (2), $310-32$ (2014). 
13. Vorhees, C. V., Skelton, M. R., Williams, M. T. Age-dependent effects of neonatal methamphetamine exposure on spatial learning. Behavioural Pharmacology. 18 (5-6), 549-562 (2007).

14. livonen, H., Nurminen, L., Harri, M., Tanila, H., Puoliväli, J. Hypothermia in mice tested in Morris water maze. Behaviour Brain Research. 141 (2), 207-213 (2003).

15. Lin, S. Y. et al. Ozone inhibits APP/A production and improves cognition in an APP/PS1 transgenic mouse model. Neuroscience. In Press, (2019).

16. Zuo, Y. et al. Preoperative vitamin-rich carbohydrate loading alleviates postoperative cognitive dysfunction in aged rats. Behavioural Brain Research. 373, 112107 (2019). 